increased transgene expression in tumors and reduced the gene expression in the liver, compared with a control vector suspension, after adenoviral vectors were injected into solid tumors in a mouse mammary carcinoma model. The median ratio of copy numbers between tumor and liver was 35.1 in the poloxamer group compared with only 0.35 in the control group, a further indication of lack of dissemination using the novel delivery system. The likely mechanism for the reduction in dissemination was considered to be the increased viscosity of the poloxamer blocking the convection of the viral vectors within the interstitial space, across the microvessel wall, and in the lumen of tumor microvessels in the injection area.

Based on these promising results in an animal model, the authors conclude that poloxamer has potential for use in the clinical setting to enhance efficacy and reduce toxicity in viral gene therapy.

Carol Lovegrove

Original article Wang Y et al. (2005) A novel method for viral gene delivery in solid tumors. Cancer Res 65: 7541-7545

\section{P-glycoprotein expression predicts response to NACT in breast cancer}

Neoadjuvant chemotherapy (NACT) is an important aspect of the management of locally advanced breast cancer, but chemoresistance can limit efficacy. The ability to predict response would allow patient-specific tailoring of treatment regimens and possibly allow the avoidance of toxicity associated with ineffective chemotherapy.

The expression of p-glycoprotein, a $170 \mathrm{kDa}$ membrane glycoprotein encoded by the multidrug resistance 1 (MDR1) gene, has been associated with the development of resistance to chemotherapy. Chintamani and colleagues evaluated the expression of p-glycoprotein in 50 patients with advanced breast cancer to determine whether pretreatment expression could be used to reliably predict response to NACT (3 cycles of cyclophosphamide $600 \mathrm{mg} / \mathrm{m}^{2}$, adriamycin $50 \mathrm{mg} / \mathrm{m}^{2}$ and 5 -fluorouracil $600 \mathrm{mg} / \mathrm{m}^{2}$ every 3 weeks). Twenty-six patients (52\%) were p-glycoprotein positive. A statistically significant negative correlation was found between clinical response and p-glycoprotein expression $(P=0.05)$. Thirty patients $(60 \%)$ showed a clinical response to NACT, and of these, 21 (70\%) were p-glycoprotein negative. Seven of nine p-glycoprotein-positive patients who responded to NACT showed only very low levels of expression. Post-NACT increases in $\mathrm{p}$-glycoprotein levels increased the number of p-glycoprotein-positive patients to $72.5 \%$, and the authors suggest that this might reflect development of acquired chemoresistance.

Overall, pre-NACT p-glycoprotein expression is associated with poor response to NACT. In addition, chemotherapy-induced increases in p-glycoprotein levels might be an indication of the development of chemoresistance and could serve as an intermediate endpoint in assessing drug response.

Carol Lovegrove

Original article Chintamani et al. (2005) Role of p-glycoprotein expression in predicting response to neoadjuvant chemotherapy in breast cancer-a prospective clinical study. World J Surg Onc 3: 61 [doi: 10.1186/ 1477-7819-3-61]

\section{Long-term use of aspirin and NSAIDs reduces the risk of colorectal cancer}

EBM A prospective cohort study involving 82,911 female participants has shown that regular long-term use of aspirin and other nonsteroidal antiinflammatory drugs (NSAIDs) reduces the risk of colorectal cancer.

The study population was recruited from the Nurses' Health Study, a study established in 1976 that enrolled US female registered nurses. Every 2 years, questionnaires were sent to the participants asking about their medicine use, including that of aspirin and other NSAIDs. Information specifically about cyclo-oxygenase 2 inhibitors was not collected.

Colorectal cancer was documented in 962 of the participants. Long-term regular aspirin use ( 2 or more standard tablets per week) was associated with a significant reduction in risk of colorectal cancer. The apparent benefit associated with aspirin use was substantially greater with increasing aspirin dose, and the greatest risk reduction was observed in women taking more than 14 doses of aspirin per week. 